\title{
WEAK APPROXIMATION FOR GENERAL DEGREE TWO DEL PEZZO SURFACES
}

\author{
AMANDA KNECHT \\ (Communicated by Ted Chinburg)
}

\begin{abstract}
We address weak approximation for certain del Pezzo surfaces defined over the function field of a curve. We study the rational connectivity of the smooth locus of degree two del Pezzo surfaces with two A1 singularities in order to prove weak approximation for degree two del Pezzo surfaces with square-free discriminant.
\end{abstract}

\section{INTRODUCTION}

A standard question in Arithmetic Geometry is, "Does a variety $X$ defined over a number field $K$ contain $K$-rational points, and if so, how are these points distributed on $X$ ?" We say that $X$ satisfies weak approximation if for any finite set of places of $K$ and points of $X$ over the completion of $K$ at these places, there exists a $K$-rational point of $X$ which is arbitrarily close to these points. In this paper, we study varieties defined over the function field of a smooth curve instead of a number field. In this context, rational points correspond to sections of fibrations over a curve, and proving weak approximation corresponds to finding sections with prescribed jet data in a finite number of fibers.

The existence of sections of rationally connected fibrations was proven by Graber, Harris and Starr [5]. Kollár, Miyaoka, and Mori proved the existence of sections through a finite set of prescribed points in smooth fibers [11, IV.6.10] and [12, 2.13]. The existence of sections with prescribed finite jet data through smooth fibers, i.e. weak approximation at places of good reduction, was proven by Hassett and Tschinkel [7. In the same paper, Hassett and Tschinkel conjectured that a smooth rationally connected variety $X$ defined over the function field of a smooth curve satisfies weak approximation even at places of bad reduction.

There are a few cases where weak approximation over the function field of a curve is known to hold at all places. Colliot-Thélène and Gille proved that weak approximation holds for stably rational varieties, connected linear algebraic groups and homogeneous spaces for these groups, and homogeneous space fibrations over varieties that satisfy weak approximation 3. In particular they proved that conic bundles over $\mathbb{P}^{1}$ and del Pezzo surfaces of degree at least four satisfy weak approximation at all places. The cases of del Pezzo surfaces of degree less than four are still open. It is known that cubic surfaces with square-free discriminant satisfy

Received by the editors January 19, 2009 and, in revised form, July 22, 2011.

2010 Mathematics Subject Classification. Primary 14M22, 14 G05.

The author was supported by the National Science Foundation under Grants 0134259 and 0240058 . 
weak approximation even at places of bad reduction [6]. This paper addresses weak approximation at places of bad reduction for degree 2 del Pezzo surfaces.

Let $k$ be an algebraically closed field of characteristic zero, $B$ a smooth curve over $k$ with function field $K=k(B)$, and $\bar{B}$ a smooth projective model of $K$ with $S:=\bar{B} \backslash B$.

Theorem 1.1. Let $X$ be a smooth degree two del Pezzo surface over $K$, and $\pi$ : $\mathcal{X} \rightarrow B$ a proper model of $X$ (i.e. an algebraic space flat over $B$ with generic fiber $X)$. Suppose the singular fibers of $\pi$ are degree two log del Pezzo surfaces with at most two $A_{1}$ singularities. Let $\mathcal{X}^{s m}$ be the locus where $\pi$ is smooth. If there exists a section $s: B \rightarrow \mathcal{X}^{s m}$, then the sections of $\mathcal{X}^{s m} \rightarrow B$ satisfy approximation away from $S$.

The proof of Theorem 1.1 relies on approximation results of [6] and extending the rational connectivity results of $[9$ to show that if $X$ is a degree two log del Pezzo surface with two $A_{1}$ singularities, then each smooth point of $X$ is contained in a free proper rational curve contained in the smooth locus of $X$. This proof also shows that the sections of $\mathcal{X}^{s m} \rightarrow B$ satisfy approximation away from $S$ when the singular fibers are degree two del Pezzo surfaces with the following singularity types:

$$
A_{2}, 2 A_{2}, A_{2}+A_{1}, A_{3}, A_{3}+A_{2}, A_{3}+A_{1}, A_{4}, A_{4}+A_{1}, A_{4}+A_{2}, A_{5}, A_{6}, D_{4} .
$$

The proofs of these cases are similar to those found below, and the details are included in [10]. Also included in [10] is the proof that a degree 2 del Pezzo surface with only $A_{1}$ singularities is simply rationally connected, but the arguments used to proved the cases of three, four, five and six $A_{1}$ singularities are very different from those found in this paper.

The rationality of $X$ and properness of $\pi$ imply the existence of sections of $\pi[5]$. When the model is regular, all sections of $\pi$ are contained in the smooth locus, so we find:

Corollary 1.2. Let $X$ be a smooth degree two del Pezzo surface over $K$. If $X$ admits a regular proper model $\pi: \mathcal{X} \rightarrow B$ whose singular fibers are degree two log del Pezzo surfaces with two $A_{1}$ singularities, then weak approximation holds for $X$ away from $S$.

This corollary is applicable whenever $B$ is a smooth projective curve because $X$ will always admit a regular proper model. There exist smooth degree two del Pezzo surfaces that admit models with worse than $A_{1}$ singularities. For example the family

$$
\left\{x^{3} y-x y^{3}+t z^{4}=w^{2}\right\} \subset \mathbb{P}(1,1,1,2) \times \mathbb{A}_{t}^{1}
$$

over the $t$-line has a fiber with worse than even rational double points when $t=0$. Nevertheless, Corollary 1.2 says that weak approximation holds for 'generic' degree two del Pezzo surfaces.

Corollary 1.3. Suppose $X$ is a smooth degree two del Pezzo surface defined over a smooth curve $B$. If the discriminant of $X$ is square-free, then sections of $f: \mathcal{X} \rightarrow B$ satisfy approximation away from $S$.

The discriminant of a smooth degree two del Pezzo surface is discussed in the next section. 
Shortly after we posted our paper on the arXiv preprint server (arXiv:0809.1251), Chenyang Xu posted a paper generalizing Theorem 6.1. A revised version of Xu's manuscript will soon appear in print [15. Xu was also able to prove weak approximation for generic degree 1 del Pezzo surfaces in a subsequent paper [14].

\section{DiscRiminants}

Let $X$ be a normal projective surface of degree 2 over $k$ with ample anti-canonical sheaf $\omega_{X}^{-1}$. Furthermore, suppose that the singularities of $X$ are at most rational double points. We call $X$ a degree 2 log del Pezzo surface with rational double points. Such an $X$ is a double cover of $\mathbb{P}^{2}$ branched over a quartic curve without multiple components, which may be thought of as a quartic hypersurface in the weighted projective space $\mathbb{P}(1,1,1,2)$ defined by the equation $w^{2}=f_{4}(x, y, z)$, where $f_{4} \in k[x, y, z]_{4}[\underline{8}$.

Assume that $X$ is a smooth degree two del Pezzo surface defined over a smooth curve $B$, and $f: \mathcal{X} \rightarrow B$ is a model of $X$ with smooth total space. We would like to define the discriminant for this model, so we replace the singular space $\mathbb{P}(1,1,1,2)$ with its minimal resolution $W$. Now the fibers of $f$ are degree four hypersurfaces in $W$. Suppose $Y$ is a degree four hypersurface in $\mathbb{P}(1,1,1,2)$ and contains $[0,0,0,1]$. Let $\widetilde{Y}$ be the proper transform of $Y$ in $W$. Then $\widetilde{Y}$ will intersect the exceptional $\mathbb{P}^{2}$ along a conic of self-intersection -4 .

Let $d f: \mathcal{T}_{\mathcal{X}} \rightarrow f^{*} \mathcal{T}_{B}$ be the differential map from a rank three vector bundle to a rank one vector bundle over $\mathcal{X}$. Let $D(d f)$ be the degeneracy locus of $d f$ in $\mathcal{X}$ and define the discriminant $\Delta$ of $X$ to be the image of $D(d f)$ in $B$. The expected dimension of $D(d f)$ is zero, but the cone point $[0,0,0,1] \in \mathbb{P}(1,1,1,2)$ can lead to components of dimension one as seen in the previous paragraph. The excess intersection formula [4, 6.3] tells us that the one-dimensional components of $D(d f)$ have multiplicity two in $\Delta$. We also know that in the multiplicity of $\Delta$ at each zero-dimensional component of $D(d f)$ is the sum of the Milnor numbers of the singularities in the corresponding fiber [4, 7.1.14]. We say that the discriminant of $X$ is square-free if each component has multiplicity one, i.e. the fibers of $f: \mathcal{X} \rightarrow B$ each have at most one $A_{1}$ singularity.

\section{WEAK APPROXIMATION}

Let $K$ be a number field or the function field of a curve $B$. Let $S$ be a finite set of places of $K$ that contains the Archimedean places. For each place $\nu$ of $K$, let $K_{\nu}$ denote the $\nu$-adic completion of $K$. Let $X$ be an algebraic variety defined over $K$ and $X(K)$ the set of $K$-rational points. One says that weak approximation holds for $X$ away from $S$ if for any finite set of places $\left\{\nu_{i}\right\}_{i \in I}$ of $K, \nu_{i} \notin S$ for $i \in I$, and $\nu_{i}$-adic open subsets $U_{i} \subset X\left(K_{\nu_{i}}\right)$, there is a rational point $x \in X(K)$ such that its image in each $X\left(K_{\nu_{i}}\right)$ is contained in $U_{i}$.

By restricting ourselves to the case of function fields, we can formulate a more geometric description of weak approximation. Let $B$ be a smooth curve over an algebraically closed field of characteristic zero with function field $K=k(B)$. Let $\bar{B}$ be a smooth projective model of $K$ and set $S=\bar{B} \backslash B$. Let $X$ be a smooth proper variety over $K, \pi: \mathcal{X} \rightarrow B$ a proper flat model (existence was proven in [13]), and $X^{s m}$ the smooth locus of $\pi$. Since $\pi$ is a proper morphism, sections $s: B \rightarrow \mathcal{X}$ of $\pi$ correspond to $K$-rational points of $X$. The analogue of the finite set containing 
the Archimedean places is the set $S=\bar{B} \backslash B$, and local points are analogous to the $N$-jets defined below.

Definition 3.1. Let $\pi: \mathcal{X} \rightarrow B$ be a proper model of $X$ over $B$ :

- An admissible section of $\pi$ is a section $s: B \rightarrow \mathcal{X}^{s m}$.

- An admissible $N$-jet of $\pi$ at $b \in B$ is a section of

$$
\mathcal{X}^{s m} \times{ }_{B} \operatorname{Spec}\left(\mathcal{O}_{B, b} / \mathfrak{m}_{B, b}^{N+1}\right) \rightarrow \operatorname{Spec}\left(\mathcal{O}_{B, b} / \mathfrak{m}_{B, b}^{N+1}\right)
$$

whose image is a smooth point of $\mathcal{X}_{b}$.

- An approximable $N$-jet of $\pi$ at $b \in B$ is a section of

$$
\mathcal{X} \times{ }_{B} \operatorname{Spec}\left(\mathcal{O}_{B, b} / \mathfrak{m}_{B, b}^{N+1}\right) \rightarrow \operatorname{Spec}\left(\mathcal{O}_{B, b} / \mathfrak{m}_{B, b}^{N+1}\right)
$$

that may be lifted to a section of $\hat{\mathcal{X}}_{b} \rightarrow \hat{B}_{b}$, where $\hat{B}_{b}:=\operatorname{Spec}\left(\hat{\mathcal{O}}_{B, b}\right)$ and $\hat{\mathcal{X}}_{b}:=\mathcal{X} \times_{B} \hat{B}_{b}$.

Note that Hensel's Lemma implies that every admissible $N$-jet is approximable, and every section is admissible when the model is regular. We can now formulate a geometric notion of weak approximation:

Definition 3.2. We say that $X$ satisfies weak approximation away from $S$ if any finite collection of approximable jets of $\pi$ can be realized by a section $s: B \rightarrow \mathcal{X}$. If $\mathcal{X}$ is a regular model, this is equivalent to the condition that any collection of admissible jets of $\pi$ can be realized by a section $s: B \rightarrow X^{s m}$.

When we want to refer to a specific model $\pi: \mathcal{X} \rightarrow B$, we say that sections of $\pi$ satisfy approximation away from $S$ if any finite collection of approximable jets of $\pi$ can be realized by a section $s: B \rightarrow \mathcal{X}$.

Precisely, weak approximation holds if for every finite collection $\left(b_{i}, \ldots, b_{k}\right)$ of distinct closed points in $B$ and every collection $\left(s_{1}, \ldots, s_{k}\right)$ of formal power series sections of $\pi$ over $b_{i}$ such that $s_{i}\left(b_{i}\right) \in \mathcal{X}_{b_{i}}^{s m}$, and for every positive integer $N$, there exists a regular section $s$ of $\pi$ which is congruent to $s_{i}$ modulo $\mathfrak{m}_{B, b_{i}}^{N+1}$.

\section{Notions OF RATiOnAL CONNECTIVITY}

Definition 4.1. A variety $X$ is rationally connected if there is a family of proper algebraic curves $g: U \rightarrow Y$ whose fibers are irreducible rational curves and a cycle morphism $u: U \rightarrow X$ such that

$$
u^{2}: U \times{ }_{Y} U \longrightarrow X \times X
$$

is dominant.

When $X$ is defined over an uncountable algebraically closed field $k$, rational connectivity is equivalent to the condition that any two very general points in $X(k)$ can be joined by an irreducible projective rational curve contained in $X[11$, IV.3.6].

Definition 4.2. Let $X$ be a smooth algebraic variety and $f: \mathbb{P}^{1} \rightarrow X$ a nonconstant morphism, so we have an isomorphism

$$
f^{*} \mathcal{T}_{X} \simeq \mathcal{O}_{\mathbb{P}^{1}}\left(a_{1}\right) \oplus \cdots \oplus \mathcal{O}_{\mathbb{P}^{1}}\left(a_{\operatorname{dim}(X)}\right)
$$

for suitable integers $a_{1}, \ldots, a_{\operatorname{dim}(X)}$. Then $f$ is free (resp. very free) if each $a_{i} \geq 0$ (resp. $a_{i} \geq 1$ ). 
When $X$ is a smooth variety over an algebraically closed field, being rationally connected is equivalent to containing one very free curve [11, IV.3.7]. Suppose we are in this situation. Then there exists a unique largest nonempty subset $X^{0} \subset X$ such that for any finite collection of distinct points in $X$ there is a very free rational curve contained in $X^{0}$ which contains these points as smooth points. Moreover, any rational curve that meets $X^{0}$ is contained in $X^{0}$ [11, IV.3.9.4]. There are no known examples where $X \neq X^{0}$. The case of $X=X^{0}$ leads to the following definition.

Definition $4.3([6,14])$. A smooth rationally connected variety $X$ is strongly rationally connected if the following equivalent conditions hold:

(1) for each point $x \in X$, there exists a rational curve $f: \mathbb{P}^{1} \rightarrow X$ joining $x$ and a generic point in $X$;

(2) for each point $x \in X$, there exists a very free rational curve containing $x$;

(3) for any finite collection of points $x_{1}, \ldots, x_{m} \in X$, there exists a very free rational curve containing the $x_{j}$ as smooth points;

(4) for any finite collection of jets

$$
j_{N, i} \in \operatorname{Spec} \frac{k[\varepsilon]}{\left\langle\varepsilon^{N+1}\right\rangle} \subset X, \quad i=1, \ldots, m
$$

supported at distinct points $x_{1}, \ldots, x_{m}$, there exists a very free rational curve smooth at $x_{1}, \ldots, x_{m}$ and containing the prescribed jets.

We note here that in Definition 14 of [6] property (2) reads 'free' and not 'very free', but having a free curve through every point has not been proven to be equivalent to any of the other conditions. When the variety $X$ is a surface, the words 'very free' can be replaced by 'free' in property (2).

Proposition 4.4. A smooth rationally connected surface $X$ is strongly rationally connected if and only if for each point $x \in X$, there exists a free rational curve containing $x$.

Proof. When there is a family of rational curves through $x$ dominating $X$, one of these curves is guaranteed to be free [12, 1.1]. Now suppose there is a free curve through every point in $X$. For any $x \in X$, let $R$ be a free rational curve containing $x$. Let $D$ be a free curve containing the generic point. Since $R$ and $D$ are free, we know that $R^{2}, D^{2} \geq 0$. By the Hodge Index Theorem, $D^{2} R^{2}-(D . R)^{2}<0$. Thus the curves $D$ and $R$ intersect in $X$. We can smooth this curve to a rational curve joining $x$ and the generic point [11, II.7.6].

Our goal is to prove that the smooth locus of a degree two log del Pezzo surface with two $A_{1}$ singularities is strongly rationally connected because Hassett and Tschinkel proved the following theorem:

Theorem $4.5([6,15])$. Let $\pi: \mathcal{X} \rightarrow B$ be a smooth morphism whose fibers are strongly rationally connected. Assume that $\pi$ has a section. Then sections of $\mathcal{X} \rightarrow$ $B$ satisfy approximation away from $S$.

\section{Combs And free Curves}

We want to find a free rational curve through any smooth point in $X$. Suppose we have a surface containing many very free rational curves. Suppose also that we have found a rational curve through a point $x \in X$ which is not free. This section shows how we use combs to create a free rational curve through the point $x$. 
Definition 5.1. A comb with $p$ teeth is a connected and reduced curve with $p+1$ irreducible components $D, C_{1}, \ldots, C_{p}$ such that:

(1) $D$ is smooth and called the handle;

(2) every $C_{i}$ is isomorphic to $\mathbb{P}^{1}$ and these are called the teeth;

(3) the only singularities of $C$ are ordinary nodes;

(4) every $C_{i}$ intersects $D$ in a single point, and $C_{i} \cap C_{j}=\emptyset$ when $i \neq j$.

A rational comb is a comb whose handle is a smooth rational curve.

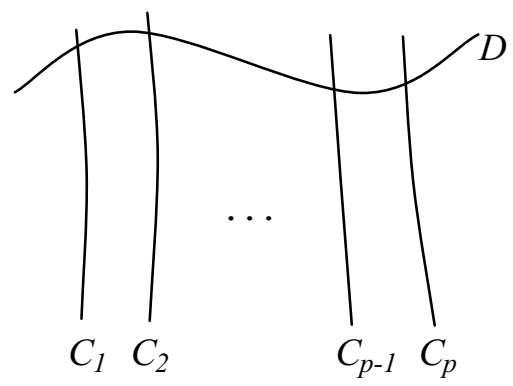

Figure 1. Comb with $p$ teeth

We construct a comb whose handle is an arbitrary nonfree rational curve $D$ on a smooth quasi-projective variety $X$. Assume there exist several very free rational curves $C_{1}, \ldots, C_{p} \subset X$ meeting $D$ at distinct smooth points $d_{i} \in D$. We want to deform the union $D \cup C_{1} \cup \ldots \cup C_{p}$ into a very free rational curve. One construction of such a comb was considered by Kollár, Miyaoka, and Mori; see [11, II.7.9, II.7.10]. A more recent construction of a comb that deforms nicely comes from the techniques of Graber, Harris, and Starr. This construction considers deformations in the Hilbert scheme of $X$. We include here some essential facts about Hilbert schemes which are used in constructing the comb that frees $D$.

There is a $k$-scheme $\operatorname{Hilb}(X)$ parameterizing closed subschemes of $X$. Assume $X$ is smooth, and let $Z$ be a closed subscheme of $X$ given by the ideal sheaf $\mathscr{I}_{Z}$. Assume $Z$ is a local complete intersection; i.e. $\mathscr{I}_{Z}$ is locally generated by $\operatorname{codim}(Z, X)$ elements. Then $\mathscr{I}_{Z} / \mathscr{I}_{Z}^{2}$ is locally free on $Z$ and its dual is the normal bundle $\mathcal{N}_{Z / X}$ of $Z$ in $X$. In this situation the following hold [11, I.2.8]:

1. $\mathcal{T}_{[Z]} \operatorname{Hilb}(X) \cong \mathrm{H}^{0}\left(Z, \mathcal{N}_{Z / X}\right)$.

2. If $\mathrm{H}^{1}\left(Z, \mathcal{N}_{Z / X}\right)=0$, then $\operatorname{Hilb}(X)$ is smooth at $[Z]$.

Suppose there are disjoint smooth rational curves $C_{1}, \ldots, C_{p}$ each meeting a rational curve $D$ transversely in a single point $d_{i}, i=1, \ldots, p$. Fix a point $P \in D$ that is not one of the nodes $d_{i}$. Then we can deform their union $D^{*}=D \cup C_{1} \cup$ $\ldots \cup C_{p}$ into an irreducible curve provided the following conditions hold [5, 2.6]:

1. The sheaf $\mathcal{N}_{D^{*} / X}$ is generated by global sections.

2. $\mathrm{H}^{1}\left(D^{*}, \mathcal{N}_{D^{*} / X}\right)=0$.

We can see this by considering the sequence

$$
\left.0 \rightarrow \mathcal{N}_{D / X} \rightarrow \mathcal{N}_{D^{*} / X}\right|_{D} \rightarrow Q \rightarrow 0,
$$

where $Q$ is a torsion sheaf supported at the points $d_{i}=D \cap C_{i}$. If $\mathcal{N}_{D^{*} / X}$ is generated by global sections, then we can find a global section $s \in \mathrm{H}^{0}\left(C, \mathcal{N}_{C / X}\right)$ such 
that, for each $i=1, \ldots, p$, the restriction of $s$ to a neighborhood of $d_{i}$ is not in the image of $\mathcal{N}_{D / X}$. This means that $s$ corresponds to a first-order deformation of $C$ that smoothes the nodes $d_{i}$ of $D^{*}$. If the second condition holds, there is no obstruction to lifting our first-order deformation to a global deformation of $D^{*}$ smoothing the nodes.

For a general fixed point $P \in D$, we would like to create a comb which deforms into an irreducible free curve while still passing through $P$. In order to achieve this, we replace the conditions above with new conditions:

$1^{\prime}$. The sheaf $\mathcal{N}_{D^{*} / X}(-P)$ is generated by global sections.

$2^{\prime}$. $\mathrm{H}^{1}\left(C, \mathcal{N}_{D^{*} / X}(-P)\right)=0$.

Theorem 5.2 ([1, 27]). Let $X$ be a smooth projective variety of dimension at least 3 over an algebraically closed field. Let $D \subset X$ be a smooth irreducible curve and $\mathscr{M}$ a line bundle on $D$. Let $C \subset X$ be a very free rational curve intersecting $D$ and let $\mathcal{C}$ be a family of nodal rational curves on $X$ parameterized by a neighborhood of $[C]$ in $\operatorname{Mor}\left(\mathbb{P}^{1}, X\right)$. Then there are curves $C_{1}, \ldots, C_{p} \in \mathcal{C}$ such that $D^{*}=D \cup C_{1} \cup \ldots \cup C_{p}$ is an immersed comb and satisfies the following conditions:

1. The sheaf $\mathcal{N}_{D^{*} / X}$ is generated by global sections.

2. $H^{1}\left(D^{*}, \mathcal{N}_{D^{*} / X}\left(\mathscr{M}^{*}\right)\right)=0$, where $\mathscr{M}^{*}$ is the unique line bundle on $D^{*}$ that extends $\mathscr{M}$ and has degree 0 on the $C_{i}$.

Thus on a variety of dimension at least three containing many very free curves, rational curves meeting the very free curves can be freed. We can generalize this result a little.

Proposition 5.3. Let $X$ be a smooth surface over an algebraically closed field. Let $D \subset X$ be an irreducible rational curve and $P$ a point on $D$. Let $C \subset X$ be a very free rational curve intersecting $D$ and let $\mathcal{C}$ be a family of rational curves on $X$ parameterized by a neighborhood of $[C] \in \operatorname{Mor}\left(\mathbb{P}^{1}, X\right)$. Then there are curves $C_{1}, \ldots, C_{p} \in \mathcal{C}$ such that $D^{*}=D \cup C_{1} \cup \ldots \cup C_{p}$ is a comb whose nodes can be smoothed to create a free rational curve containing $P$.

Proof. We modify the smoothness of $D$ and dimension of $X$ in Theorem 5.2 .

Step 1. Reduction to the case where $D$ is a smooth rational curve.

Suppose $D$ is singular. Then we will work with the normalization $\widetilde{D} \rightarrow D$. When considering the Hilbert Scheme, it is essential that $D$ be embedded in $X$, so we take an embedding $\widetilde{D} \hookrightarrow \mathbb{P}^{3}$ and consider the diagonal map $\Delta: \widetilde{D} \rightarrow X \times \mathbb{P}^{3}$. This is an embedding with image $D^{\prime}$ isomorphic to $\widetilde{D}$. Let $\pi_{1}$ denote the projection from $X \times \mathbb{P}^{3}$ to the first factor. Then $\pi_{1}\left(D^{\prime}\right) \simeq D$. Any moving of $D^{\prime} \subset X \times \mathbb{P}^{3}$ can be projected to $X$ to give a deformation of $D$. From now on assume $D \subset X$ is a smooth, irreducible, rational curve.

Step 2. Reduction from surfaces to three-folds.

Suppose $S$ is a smooth surface and $f: \mathbb{P}^{1} \rightarrow S$ is a morphism. Consider the three-fold $X=\mathbb{P}^{1} \times S$ and the map $g=(i d, f): \mathbb{P}^{1} \rightarrow X$. If we let $\pi_{2}: X \rightarrow S$ be the projection onto the second factor, then $f=\pi_{2} \circ g$. Since $\pi_{2}$ is smooth, the map $g^{*} \mathcal{T}_{X} \rightarrow f^{*} \mathcal{T}_{S}$ is surjective. Thus if $g^{*} \mathcal{T}_{X}$ is ample, then $f^{*} \mathcal{T}_{S}$ is ample. 
Now once we find a rational curve in $X^{s m}$ containing a given smooth point $x$, we know how to free it. So what is left to do is to find rational curves through points.

\section{Proof of the MAIN THEOREM}

Keel and McKernan proved that the smooth locus of any log del Pezzo surface is rationally connected [9]. As stated in Section 4, the main contribution made by this paper is the following refinement for degree two del Pezzos:

Theorem 6.1. Let $X$ be a degree two log del Pezzo surface with two $A_{1}$ singularities and $X^{\text {sm }}$ the smooth locus of $X$. Then $X^{s m}$ is strongly rationally connected.

Proof. Let $\varphi: X \rightarrow \mathbb{P}^{2}$ be a double cover of $\mathbb{P}^{2}$ branched over a quartic plane curve $Q$. Since $X$ has two $A_{1}$ singularities, $Q$ has two nodes $q_{1}, q_{2}$ [2, III.7.1]. In order to prove the theorem, for every point $x \in X^{s m}$ we will find a free curve $C \subset X^{s m}$ containing $x$.

Consider arbitrary $x \in X^{s m}$. Let $\varphi(x)=p \in \mathbb{P}^{2} \backslash\left\{q_{1}, q_{2}\right\}$. We must find a proper curve in $p \in \mathbb{P}^{2} \backslash\left\{q_{1}, q_{2}\right\}$ which lifts to a free rational curve in $X^{s m}$. The simplest plane curves are lines, so we can try to find lines through $p$ that lift to rational curves. By the Hurwitz formula we see that a generic line in $\mathbb{P}^{2}$ will lift to a curve of genus one in $X$. However, lines simply tangent to $Q$ will lift to rational curves. Thus, we consider lines tangent to the branch curve $Q$.

First we consider the case when $p$ is not contained in $Q$. Let $x \in X$ be such that $\varphi(x)=p \notin Q$. Let $\widetilde{Q}$ be the normalization of $Q$ and consider the projection from $\pi_{p}: \mathbb{P}^{2} \backslash\{p\} \rightarrow \mathbb{P}^{1}$. Then $\pi_{p}$ restricted to the genus two curve $\widetilde{Q}$ is a fourto-one cover of the projective line with ramification index equal to ten. Since the ramification number is ten, there are at least four lines through $p$ that are tangent to $Q$, and three of these lines will not contain the singular point $q$. We only need to pick one of these lines and analyze the four ways a line can be tangent to a quartic: one simple tangent, one three-tangent, one four-tangent, or two simple tangents.

Next suppose that $p$ is a smooth point on the quartic $Q$. If the tangent line of $Q$ at $p$ does not contain the singular point, $q$, then we can use the tangent line at $p$ to find a free curve through $x$ with the analysis below. The tangent line at $p$ passing through $q$ is not actually a problem. If we consider projection from $p$ again, we have a three-to-one cover of the line with ramification index equal to eight and are guaranteed that $p$ is contained in at least four lines tangent to $Q$. We simply pick one of the lines missing the singular point.

Now we analyze the four cases of tangency between a line and a quartic curve, and construct free curves in $X$ from lifts of the lines.

One simple tangent or inflectional 3-tangent. Suppose that we can find a line $L$ through $p$ that is simply tangent to $Q$ at a smooth point of $Q$ and intersects the quartic in two other distinct points or we can find a line $L$ through $p$ that is three-tangent to $Q$ at a smooth point. By the Hurwitz formula, we know that the normalization, $\widetilde{C}$, of the curve $C \subset X$ lying over $L$ is rational; thus $C$ is rational. Let $C$ be the image of the projective line under the map $f: \mathbb{P}^{1} \rightarrow X$.

We have an exact sequence

$$
0 \longrightarrow \mathcal{T}_{\mathbb{P} 1} \longrightarrow f^{*} \mathcal{T}_{X} \longrightarrow \mathcal{N}_{f} \longrightarrow 0 .
$$


We know that $\mathcal{T}_{\mathbb{P}^{1}}=\mathcal{O}_{\mathbb{P}^{1}}(2)$, and by the double point formula, $\operatorname{deg} \mathcal{N}_{f}=C^{2}-$ $2(\#$ nodes on $C)=2-2=0$. The extension

$$
0 \longrightarrow \mathcal{O}_{\mathbb{P}^{1}}(2) \longrightarrow f^{*} \mathcal{T}_{X} \longrightarrow \mathcal{O}_{\mathbb{P}^{1}} \longrightarrow 0
$$

is given by an element of $\operatorname{Ext}^{1}\left(\mathcal{O}_{\mathbb{P}^{1}}, \mathcal{O}_{\mathbb{P}^{1}}(2)\right)=\mathrm{H}^{1}\left(\mathbb{P}^{1}, \mathcal{O}_{\mathbb{P}^{1}}(2)\right)=0$. Thus, the sequence splits and $f^{*} \mathcal{T}_{X}=\mathcal{O}_{\mathbb{P}^{1}}(2) \oplus \mathcal{O}_{\mathbb{P}^{1}}(0)$, and $C$ is a free curve through the point $x$.

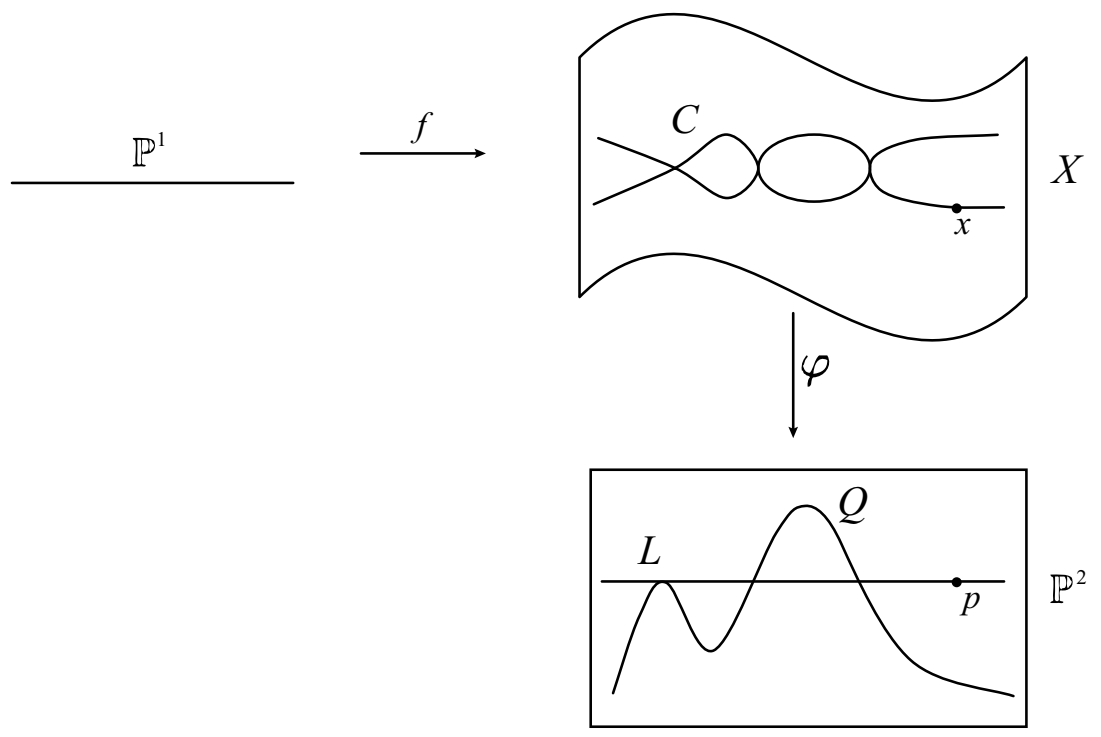

Figure 2. One simple tangent

In order to find a free curve through $x$ we will construct a comb with $C$ as the handle. Since $X^{s m}$ is rationally connected, we know that $X^{s m}$ contains a very free rational curve $D$. The Hodge Index Theorem and the fact that the $D^{2}$ and $C^{2}$ are positive imply that $D$ and $C$ intersect. By Proposition 5.3 , we see that there exists a free curve through $x$.

One inflectional 4-tangent. Suppose we have a line $L$ through $p$ intersecting the quartic $Q$ at only one smooth point. Then $L$ is four-tangent to $Q$ at the point of intersection, and the corresponding curve $C \subset X$ above $L$ will have a tacnode above the intersection. Because the arithmetic genus of the normalization of the curve $C$ is -1 , we know that $C$ must be the union of two irreducible rational curves $C_{1}, C_{2}$. Instead of considering the entire curve $C$, we shall focus on the component $C_{1}$ passing through the original point $x \in X$.

Because each $C_{i}$ is mapped one-to-one onto the line $L$ in $\mathbb{P}^{2}$ by the anti-canonical sheaf of $X$, we know that $C_{i} \cdot K_{X}=-1$. Adjunction then implies that the selfintersection, hence the degree of the normal sheaf, of each $C_{i}$ is -1 . Hence, our curve $C_{1}$ is not free.

We use the dual curve of $Q$ to show that we can use a comb as in Proposition 5.3 to free $C_{1}$. For each point $q \in Q$, let $\mathcal{T}_{q} Q$ be the tangent line to $Q$ at $q$. Consider $\mathcal{T}_{q} Q$ as a point in the dual projective plane $\left(\mathbb{P}^{2}\right)^{*}$. There is a map from $Q$ to its dual curve $Q^{*} \subset\left(\mathbb{P}^{2}\right)^{*}$ which sends a point $q$ to $\mathcal{T}_{q} Q$. The singularities of $Q^{*}$ are: nodes 
corresponding to bitangents to $Q$, cusps corresponding to inflectional 3-tangents of $Q$, and tacnodes corresponding to inflectional 4-tangents of $Q$. The dual curve to a quartic with one node is a planar curve of degree 10 and has only a finite number of singularities. Thus, for an infinite number of points on the line $L$, there are lines through these points that are simply tangent to $Q$ and intersect $L$ transversely. These lines lift to free curves in $X$ as shown above and can be made into the very free curves that we need in order to apply the proposition.

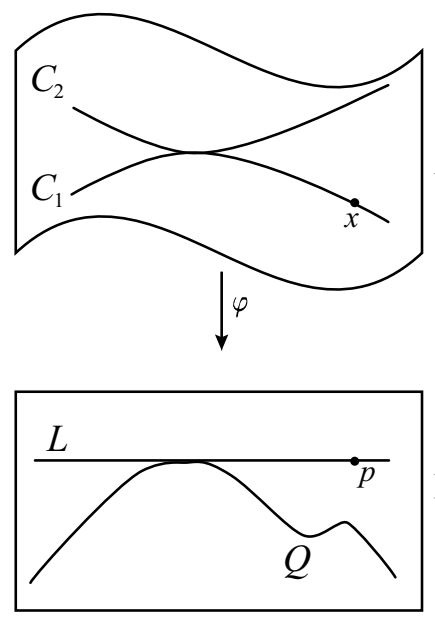

(A) Inflectional 4-Tangent
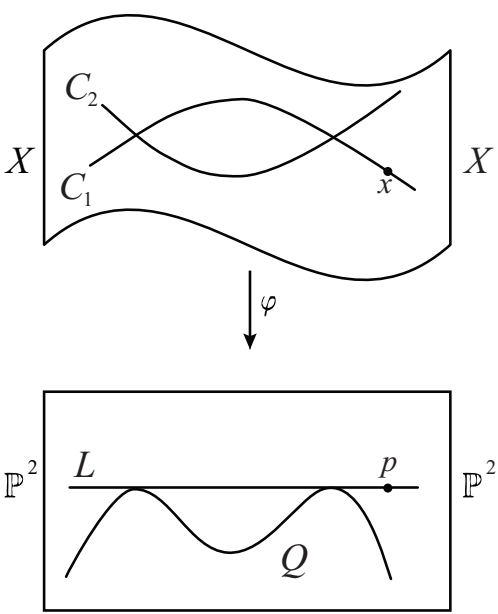

(B) Bitangent

FigURE 3

Two simple tangents. Finally suppose there exists a line $L$ through $p$ that is tangent to $Q$ at two distinct nonsingular points. Then the curve $C \subset X$ lying above $L$ will have a node above each of these tangencies. The arithmetic genus of the normalization of $C$ is -1 , so we know that $C$ is the union of two rational curves $C_{1}, C_{2}$. As in the case above, we find that the degree of the normal sheaf of each $C_{i}$ is -1 . Suppose $C_{1}$ is the component that contains the point $x$. We can use very free curves intersecting $C_{1}$ to create a comb to free $C_{1}$. The resulting curve will be a free rational curve through $x$, as desired.

As stated in the introduction, this proof can be easily modified to show that the smooth locus of a degree two del Pezzo surface is strongly rationally connected when the singularity type of the surface is one of the following [10:

$$
A_{2}, 2 A_{2}, A_{2}+A_{1}, A_{3}, A_{3}+A_{2}, A_{3}+A_{1}, A_{4}, A_{4}+A_{1}, A_{4}+A_{2}, A_{5}, A_{6}, D_{4} .
$$

\section{ACKNOWLEDGMENTS}

The author is grateful to her thesis advisor, Brendan Hassett, for the many conversations they had about this topic. She also benefitted from talking to Damiano Testa and Brad Duesler. 


\section{REFERENCES}

1. C. Araujo and J. Kollár. Rational Curves on Varieties. In Higher dimensional varieties and rational points (Budapest, 2001), volume 12 of Bolyai Soc. Math. Stud., pages 13-68, Springer, Berlin, 2003. MR2011743 (2004k:14049)

2. W. Barth, C. Peters, and A. Van de Ven. Compact Complex Surfaces, volume 4 of Ergebnisse der Math. Springer-Verlag, Berlin, 1984. MR749574 (86c:32026)

3. J.L. Colliot-Thélène and P. Gille. Remarques sur l'approximation faible sur un corps de fonctions d'une variable. In Arithmetic of higher-dimensional algebraic varieties (Palo Alto, CA, 2002), volume 226 of Progr. Math., pages 121-134, Birkhäuser Boston, Boston, MA, 2004. MR2029865 (2005h:11132)

4. W. Fulton Intersection theory, 2nd ed., Ergebnisse der Mathematik und ihrer Grenzgebiete. 3. Folge. Springer, Berlin, 1998. MR1644323(99d:14003)

5. T. Graber, J. Harris, and J. Starr. Families of rationally connected varieties. J. Amer. Math. Soc., 16(1):57-67, 2003. MR 1937199 (2003m:14081)

6. B. Hassett and Y. Tschinkel. Approximation at places of bad reduction for rationally connected varieties. Pure Appl. Math. Q., 4(3):743-766, 2008. MR.2435843 (2010h:14081)

7. Weak approximation over function fields. Invent. Math., 163(1):171-190, 2006. MR2208420 (2007b:14109)

8. F. Hidaka and K. Wantanabe. Normal Gorenstein surfaces with ample anti-canonical divisor. Tokyo J. Math., 4(2):319-330, 1981. MR646042 (83h:14031)

9. S. Keel and J. McKernan. Rational curves on quasi-projective surfaces. Mem. Amer. Math. Soc., 140(669):viii+153 pp., 1999. MR:1610249 (99m:14068)

10. A. Knecht. Weak approximation for degree 2 del Pezzo surfaces at places of bad reduction. Dissertation, Rice University. ProQuest LLC, Ann Arbor, 2007. MR2710005

11. J. Kollár. Rational curves on algebraic varieties, volume 32 of Ergebnisse der Math. SpringerVerlag, Berlin, 1996. MR1440180 (98c:14001)

12. J. Kollár, Y. Miyaoka, and S. Mori. Rationally connected varieties. J. Algebraic Geom., 1(3):429-448, 1992. MR 1158625 (93i:14014)

13. M. Nagata. A generalization of the imbedding problem of an abstract variety in a complete variety. J. Math. Kyoto Univ., 3: 89-102, 1963. MR0158892 (28:2114)

14. C. Xu. Weak Approximation for Low Degree Del Pezzo Surfaces. J. Algebraic Geom., forthcoming.

15. Strong Rational Connectedness of Surfaces. J. Reine Angew. Math., 665:189-205, 2012.

Department of Mathematics, Rice University, 6100 Main Street, Houston, Texas 77005

Current address: Department of Mathematics and Statistics, Villanova University, 800 Lancaster Avenue, Villanova, Pennsylvania 19085

E-mail address: amanda.knecht@villanova.edu 